\title{
Transphosphorylation of EGFR at Y845 plays an important role in its autophosphorylation and kinase activity
}

\author{
HUI SONG ${ }^{1,2^{*}}$, LEI HUANG ${ }^{1 *}$, MING ZHANG $^{2}$, XIUWEI WANG ${ }^{2}$, SHUANG SONG ${ }^{2}$ and LIN YANG ${ }^{1,2}$ \\ ${ }^{1}$ The Cyrus Tang Hematology Center, Soochow University, Suzhou, Jiangsu 215123; \\ ${ }^{2}$ Xi'an Jiaotong University Suzhou Academy, Suzhou, Jiangsu 215123, P.R. China
}

Received January 14, 2014; Accepted February 19, 2014

DOI: 10.3892/or.2014.3102

\begin{abstract}
Epidermal growth factor receptor (EGFR) plays a critical role in various types of human cancer such as lung, breast, brain and colon cancer. However, how EGFR activation is initiated and what type of interaction is linked to its transphosphorylation and autophosphorylation as well as the biological consequences in case of interruption and blockade of these two types of EGFR phosphorylation, remain elusive. In the present study, we provided evidence that EGFR transphosphorylation at Y845 indeed plays an important role in its autophosphorylation and kinase activity. Our results suggest that the modulation of EGFR transphosphorylation may influence its activity and function, indicating a potential target mechanism for treating EGFR-associated diseases and various types of cancer.
\end{abstract}

\section{Introduction}

Epidermal growth factor receptor (EGFR) is a membrane tyrosine kinase receptor that belongs to the EGF tyrosine kinase receptor family consisting of EGFR (ErbB1), Her2 (ErbB2), ErbB3 and ErbB4 $(1,2)$. EGFR monomer is composed of four domains (3), namely, extracellular, transmembrane, intracellular domain (including kinase domain) and cytoplasmic tail $(4,5)$. According to the literature, EGF or other growth factors can cause EGFR to change its extracellular domain structure and then trigger dimerization of intracellular domain (6-13). In response to growth factors, EGFR forms either homodimer or heterodimer with other family members, therefore it subsequently changes its protein structure and protein interaction with numerous intracellular proteins (14-17). EGFR is subject to autophosphorylation and/or transphosphorylation that are essential processes for

Correspondence to: Dr Hui Song, Xi'an Jiaotong University Suzhou Academy, 99 Ren'ai Road D101, Suzhou Industry Park, Suzhou, Jiangsu 215123, P.R. China

E-mail: sunny_um@yahoo.com

*Contributed equally

Key words: epidermal growth factor receptor, transphosphorylation, autophosphorylation, kinase activity, cancer
EGFR to become an active molecule and play important roles in regulating several signaling pathways, such as MAPK, PI3-Akt and JAK-Stat 3/5 pathways (18-20). There are several critical tyrosine phosphorylation sites in the intracellular domain of EGFR. For example, Y845, 992, 1045, 1068, 1086 and 1173. These differential phosphorylation sites correspond to different signal pathways and functions (19). Generally, Y845 is associated with the Stat signaling pathway. Y992, 1068, 1086 and 1173 are generally linked to MAPK and Akt signaling pathways (19), while Y1045 phosphorylation is associated with the interaction with cbl that regulates EGFR ubiquitination and degradation (21). However, what determines these phosphorylation sites and how an individual site is regulated over the entire process remain unclear.

Although EGFR is one of the most intensively studied receptor tyrosine kinases (RTKs), it remains elusive as to whether or not its intra-tyrosine phosphorylations are interrelated. As a cell surface molecule, EGFR plays an essential and fundamental role in dictating cell proliferation and differentiation, cell cycle control, biological development, tumorigenesis and malignant development (22-24), therefore it is necessary to extensively elucidate its activation and interplay among EGFR intracellular tyrosine phosphorylations in various cellular environments. As a traditionally accepted concept, upon ligand stimulation, EGFR forms homodimer or heterodimer with one of the other three family members in physiological conditions, which subsequently results in phosphorylation of EGFR by either Src kinase or autophosphorylation. However, previous studies demonstrated that high local EGFR concentration may force EGFR to form dimers even in the absence of ligand stimulation (14). The phosphorylated EGFR provides docking sites for binding downstream adaptor proteins and thereafter activates several downstream signaling pathways. Upon Src-induced phosphorylation at Y845 on EGFR, the phosphorylated Y845 serves as docking site to recruit Stat3/5 and result in phosphorylation of Stat 3 and/or Stat5, which are transcription factors and can form homo- or heterodimers. The dimerized Stat 3 or Stat5 translocates into the nucleus and regulates cell proliferation, differentiation, cell cycle and migration. Src-activated signal pathway through Y845 is typically considered a transphosphorylation pathway. EGF ligand stimulation also causes an autophosphorylation of EGFR. Several tyrosine residues in intracellular domain of EGFR are involved in this type of autophosphorylation such as Y992, 
1068, 1086 and 1173 and provide docking sites for adaptor proteins such as Shc, Grb2 and Gab that result in the activation of PI3K/Akt and Ras/MAPK signaling pathways (19). The activation of PI3K/Akt and /or Ras/MAPK pathways has been linked to various types of cancer $(22,25,26)$. Depending on specific cell types or environments, one of these pathways may dominate or all of these pathways equally contribute to cellular processes. Despite these advances, the interplay between transphosphorylation and autophosphorylation of EGFR remains elusive.

Here, we report that transphosphorylation of EGFR at Y845 is linked to its autophosphorylation and kinase activity. The substitution of Y845 to phenylalanine (F) significantly reduced its biological function in response to ligand stimulation, suggesting the importance of this transphosphorylation site. Taken together, our results provide insights into the activation of EGFR and may indicate a potential therapeutic target for treating various types of cancer related to aberrant EFGR expression and activation.

\section{Materials and methods}

Cell lines, antibodies and chemicals. HEK293 and MCF7 cells were cultured with Dulbecco's modified Eagle's medium (DMEM) containing 10\% fetal bovine serum (FBS) and appropriate antibiotics in an incubator with $37^{\circ} \mathrm{C}$ and $5 \% \mathrm{CO}_{2}$. Antibodies were purchased as follows: anti-EFGR (Santa Cruz Biotechnology, Santa Cruz, CA, USA); anti-phosphotyrosine (4G10; Upstate); anti-phospho-EGFR-Y845 and phospho-EGFR-Y1068 (Cell Signaling Technology Inc.). All chemicals were purchased from Sigma-Aldrich Corporation and are of analytical grade except where noted otherwise.

Immunoprecipitation and immunoblotting. The cells were lysed in RIPA buffer containing $2 \mathrm{mM}$ PMSF, $2 \mathrm{mM} \mathrm{Na}_{3} \mathrm{VO}_{4}$, $2 \mathrm{mM} \mathrm{NaF}, 1 \mu \mathrm{g} / \mathrm{ml}$ aprotinin, leupeptin and pepstatin, respectively. For immunoprecipitation, a total of $500 \mu \mathrm{g}$ of cell lysate was used and diluted in $500 \mu \mathrm{l}$ of RIPA buffer with the corresponding antibodies. After addition of $2 \mu \mathrm{g}$ EGFR or flag antibody, the lysate was incubated with gentle rotation for $2 \mathrm{~h}$ at $4^{\circ} \mathrm{C}$, and then $80 \mu \mathrm{l}$ of protein-A and $20 \mu \mathrm{l}$ of protein-G beads were added into the mixture and incubated for another $2 \mathrm{~h}$. For immunoblotting, a total of $20 \mu \mathrm{g}$ of cell lysate was directly loaded and resolved onto $8 \%$ SDS-polyacrylamide gels and then probed with antibodies as indicated in the images.

In vitro kinase assay. After immunoprecipitation, the beads were washed twice using RIPA buffer containing appropriate inhibitors. After removing wash buffer from last step wash, we added $50 \mu 12 \mathrm{X}$ kinase buffer containing $100 \mathrm{mM}$ HEPES (pH 7.2), $20 \mathrm{mM} \mathrm{MgCl} 2,20 \mathrm{mM} \mathrm{MnCl}{ }_{2}, 6 \mathrm{mM} \mathrm{Na}_{3} \mathrm{VO}_{4}$, and $2.5 \mathrm{mM}$ DTT, and $0.5 \mu 1 \gamma^{32} \mathrm{p}$-ATP, mixed the reaction mixture well with beads and incubated in a $30^{\circ} \mathrm{C}$ water bath for $25 \mathrm{~min}$, then added $20 \mu \mathrm{l} 6 \mathrm{X}$ loading buffer followed by heating of the samples for $5 \mathrm{~min}$ at $100^{\circ} \mathrm{C}$. We briefly centrifuged the samples and the supernatants were used for analysis.

For in vitro transphosphorylation assay, after immunoprecipitating flag tagged EGFR-K721A with an anti-flag antibody from 1,000 $\mu \mathrm{g}$ HEK293 cell lysate and washing twice with
RIPA buffer, we added $50 \mu 12 \mathrm{X}$ kinase buffer containing $100 \mathrm{mM}$ HEPES (pH 7.2), $20 \mathrm{mM} \mathrm{MgCl}$, $20 \mathrm{mM} \mathrm{MnCl}_{2}$, $6 \mathrm{mM} \mathrm{Na} \mathrm{VO}_{4}$ and $2.5 \mathrm{mM}$ DTT and $0.5 \mathrm{mM}$ ATP and then mixed with 10, 30 and $100 \mu \mathrm{g}$ cell lysates from HEK293 cells expressing myc-tagged either wt-EGFR or EGFR-Y845F or K721A mutant, respectively. After incubation in a $30^{\circ} \mathrm{C}$ water bath for $25 \mathrm{~min}$, we washed the beads twice using RIPA buffer, then added $20 \mu \mathrm{l} 6 \mathrm{X}$ loading buffer followed by heating of the samples for $5 \mathrm{~min}$ at $100^{\circ} \mathrm{C}$. The final elutants were resolved onto $8 \%$ SDS-ployacrylamide gels and then probed with antiphospho antibodies as indicated in the images.

Transfection and establishment of stably expressing EGFR cell clones. Transfection of wild-type EGFR (wt-EGFR) or EGFR-Y845F or EGFR-Y721A was performed using Lipofectamine 2000 (Life Technologies Corporation) according to the standard procedures and the manufacturer's instructions. For establishing stable clones expressing EGFR or mutants, after $48 \mathrm{~h}$ transfection, the cells were selected with $6 \mu \mathrm{g} / \mathrm{ml}$ blasticidin (Life Technologies Corporation) for 3 weeks and single clones were isolated, and continued to culture for another 2-3 weeks in the presence of blasticidin. The cloned cells were analyzed for EGFR expression and other analysis.

Cell growth assay. Cells $\left(5 \times 10^{4}\right)$ in $1 \mathrm{ml}$ DMEM were seeded in 6-well plates. The cells were cultured in the absence or presence of EGF and cell number was counted by addition of trypan blue at time points indicated in the image. All results are in triplicate from three independent experiments.

In vitro DNA incorporation assay. For measuring cell DNA synthesis, $1 \times 10^{5} \mathrm{MCF} 7$ cells stably expressing either wt-EGFR or EGFR-Y845F mutant were seeded in 6-well plates. After $30 \mathrm{~h}$ serum starvation, the cells were treated with $30 \mu \mathrm{M}$ BrdU (BD transduction) in 10\% FBS fresh medium with or without $25 \mathrm{ng} / \mathrm{ml}$ EGF for $16 \mathrm{~h}$, then an anti-BrdU monoclonal antibody (Upstate) was used to detect incorporated BrdU substrate in the cells. An FITC-conjugated goat anti-mouse IgG antibody (Johnson Laboratory, USA) was used to amplify signals. Then, DNA incorporated cells were counted by using a fluorescence microscope. At least three independent experiments were performed as each experiment was set up in triplicate.

In vivo mammary fat pad tumor cell inoculation. MCF7 $\left(5 \times 10^{6}\right)$ cells stably expressing either wt-EGFR or EGFR-Y845F mutant were injected into the fat pads of nude mice. The tumor size was measured once per week. All animal handling procedures were performed in accordance with the institutional policies and regulations.

Flow cytometry analysis. Either wt-EGFR or EGFR-Y845F was transiently expressed in HEK293 cells. The cells were incubated with an anti-EGFR antibody for $1 \mathrm{~h}$ on ice, followed by incubation with an FITC labeled anti-IgG antibody for $30 \mathrm{~min}$ on ice, and then subjected to flow cytometry analysis.

Statistical analysis. All data are described as means \pm standard deviation. Statistical analyses were performed by Student's $\mathrm{t}$-test analysis. $\mathrm{P}<0.05$ was considered to indicate a statistically significant difference. 
A

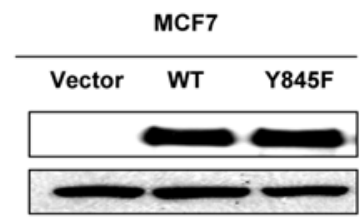

C
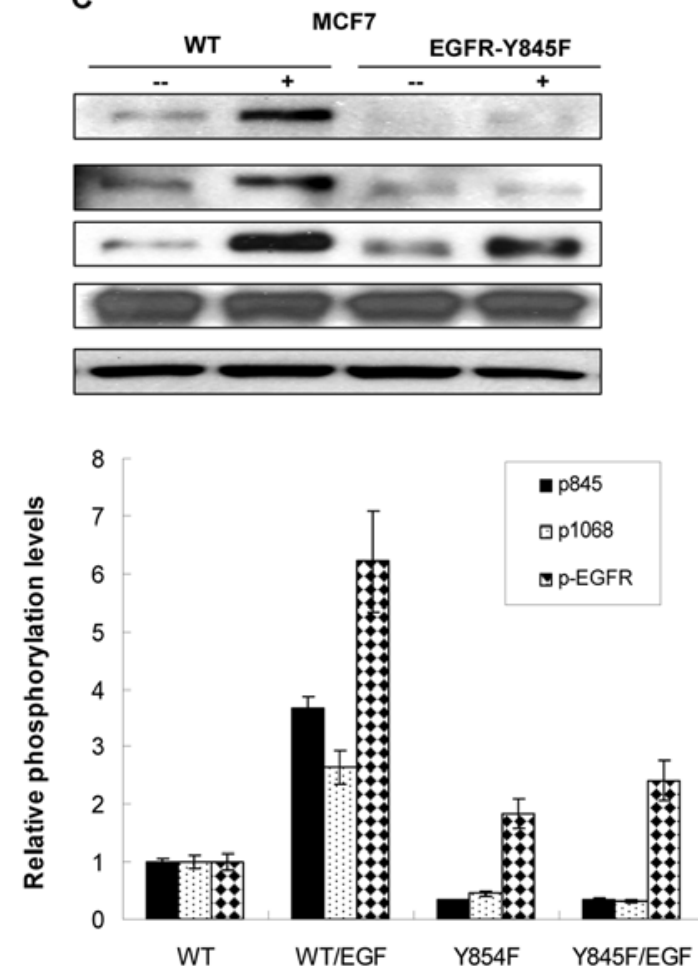

B

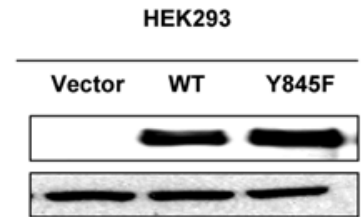

D

EGF
p-EGFR ${ }^{\text {Y845 }}$
p-EGFR ${ }^{\text {Y1068 }}$
p-E GFR (4G10)
EGFR
Actin
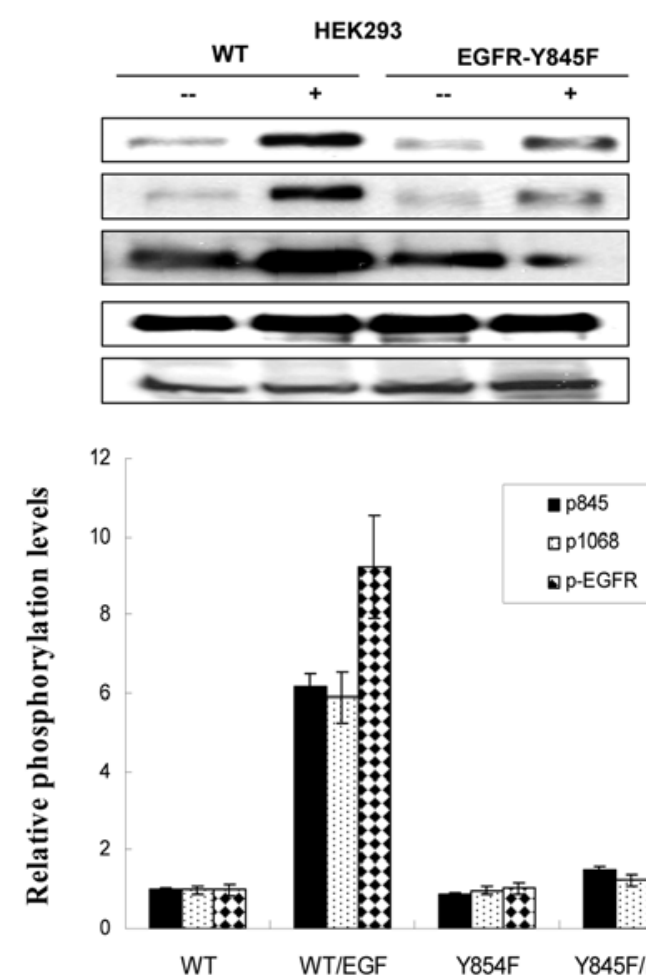

- $\mathrm{p} 845$

口p1068

๑p-EGFR

Figure 1. Phosphorylation of EGFR at Y845 regulates its autophosphorylation. A total of $20 \mu \mathrm{g}$ cell lysate was immunoblotted with antibodies as indicated. (A) Expression of wt-EGFR or EGFR-Y845F in the stably cloned MCF7 cell line. (B) Expression of wt-EGFR or EGFR-Y845F in the stably cloned HEK293 cell line. (C) Phosphorylation of EGFR in the absence or presence of EGF in MCF7 clone cells. (D) Phosphorylation of EGFR in the absence or presence of EGF in HEK293 clone cells.
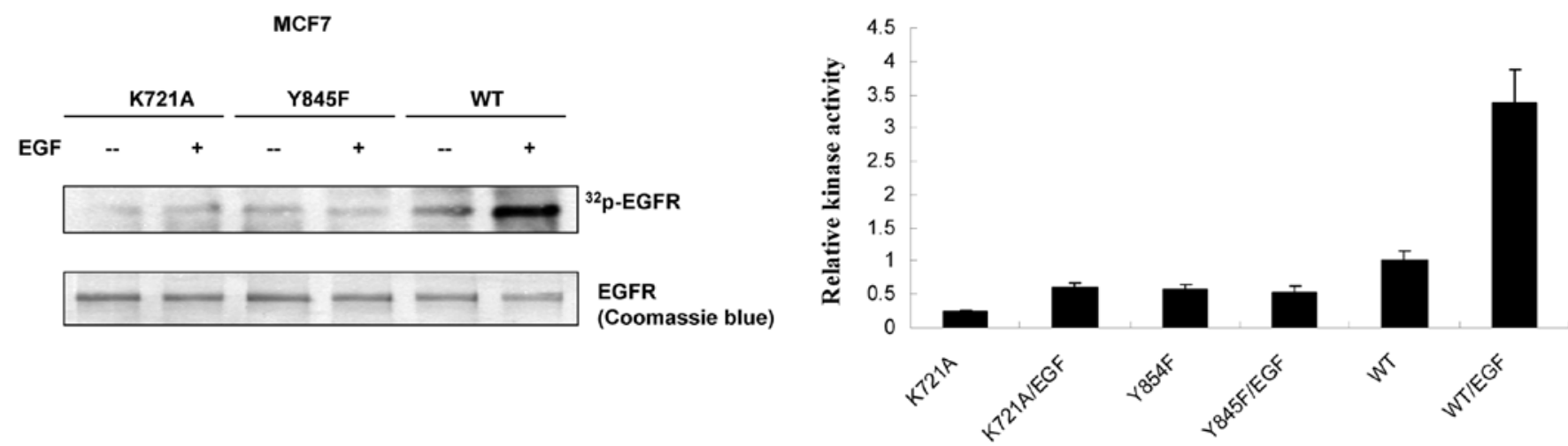

Figure 2. The EGFR-Y845F mutant reduces its EGF-induced kinase activity. A total of $500 \mu \mathrm{g}$ cell lysate from MCF7 stably cloned cells was immunoprecipitated with an EGFR antibody. Then, in vitro kinase assay was performed by addition of $\gamma-{ }^{32} \mathrm{p}$-ATP, as described in Materials and methods. The results represent an average of three independent experiments.

\section{Results}

Transphosphorylation of EGFR at Y845 affects its autophosphorylation. To determine if there is a correlation between EGFR transphosphorylation and autophosphorylation, we established stable cell clones that express either wild-type EGFR (wt-EGFR) or EGFR-Y845F mutant in an MCF7 breast carcinoma and an HEK293 cell line. Expression of target protein in both cell lines was confirmed by western blotting (Fig. 1A and B). To test if transphosphorylation deficient EGFR-Y845F has an impact on its autophosphorylation, the cloned cells were serum starved for $24 \mathrm{~h}$ and then stimulated by addition of EGF. The results demonstrated that in the absence of EGF the levels of autophosphorylation at Y1068 

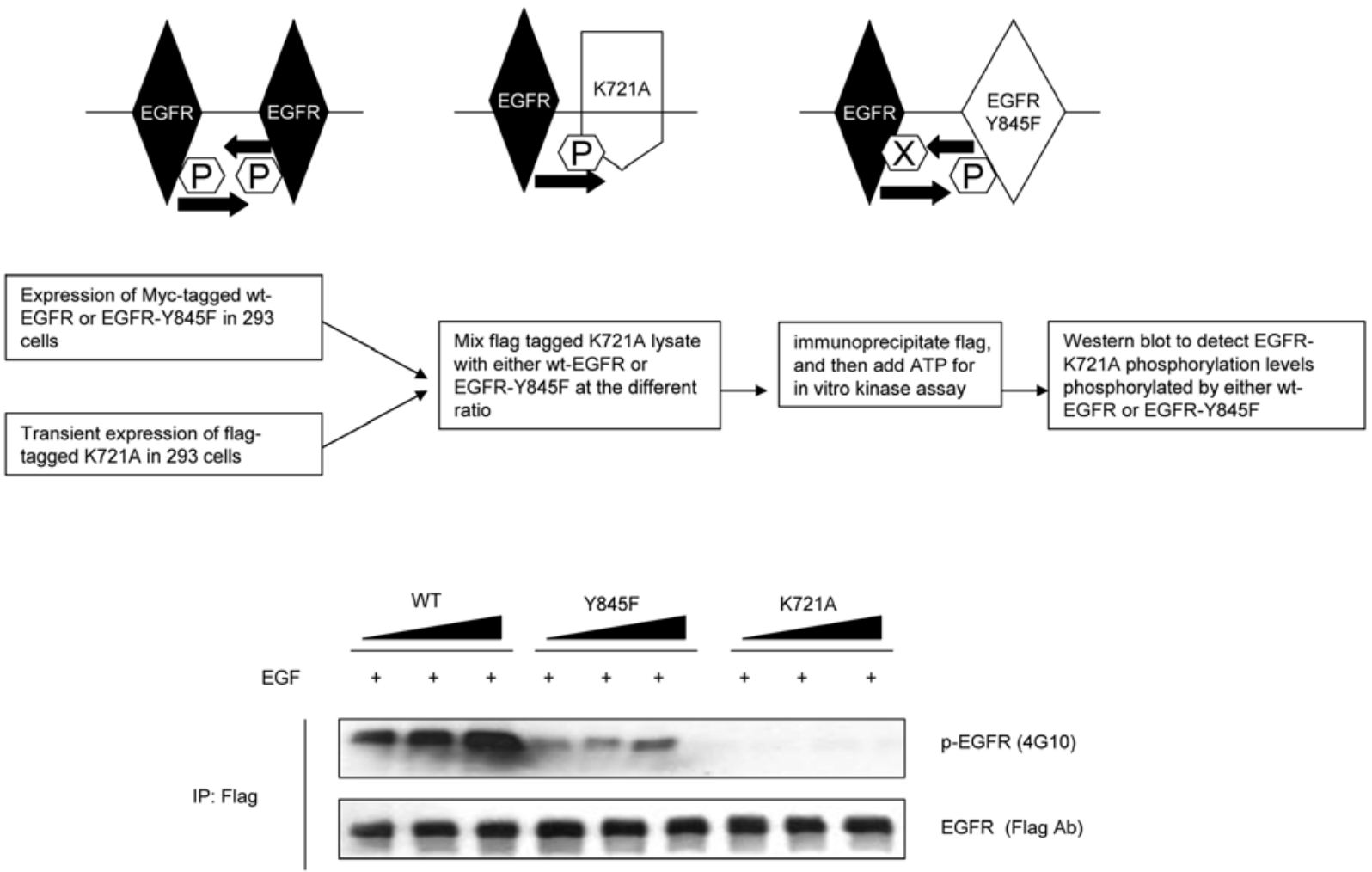

Figure 3. The EGFR-Y845F mutant reduces its ability to phosphorylate EGFR dimeric partners. A total of 1,000 $\mu \mathrm{g}$ cell lysate from transiently transfected HEK293 cells, that expressed flag tagged EGFR-K721A, was mixed with different amounts of lysate containing either Myc tagged wt-EGFR, EGFR-Y845F or EGFR-K721A, expressed in HEK293 cells and induced by EGF 30 min before harvesting the cells. The remaining assays were performed as described in Materials and methods.

and total phosphorylation were close to each other in both cell clones. However, in the presence of EGF, the phosphorylation at Y1068 and total phosphorylation were significantly reduced by EGFR-Y845F mutant, suggesting that EGFR-Y845 phosphorylation is important for its EGF-induced autophosphorylation (Fig. 1C and D).

The transphosphorylation deficient EGFR-Y845F mutant reduces its ligand-induced kinase activity. To determine if hindrance of EGF-induced phosphorylation by EGFR-Y845F mutant links to mitigation of its kinase activity, in vitro kinase assay was performed as described in Materials and methods. MCF7 clone cells were treated with EGF before harvesting. In comparison to wt-EGFR, EGFR-Y845F significantly reduced its EGF-induced kinase activity (Fig. 2), suggesting that loss of EGF-induced transphosphorylation indeed reduced its kinase activity in EGFR-Y845F mutant.

The transphosphorylation deficient EGFR-Y845F mutant reduces its ability to phosphorylate its dimeric partners. Upon the initiation of its activity, EGFR autophosphorylation occurs by cross phosphorylating its dimeric counterparts. To further distinguish if the loss of EGFR autophosphorylation in EGFR-Y845F mutant was the consequence of the loss of its transphosphorylation, we performed in vitro kinase assay by mixing different amounts of either wt-EGFR or EGFR-Y845F mutant with flag tagged EGFR-K721A, which is a kinase dead mutant and loses its autophosphorylation ability to its dimeric counterparts (Fig. 3). We were thus able to discriminate if
EGFR-Y845F mutant may reduce its auto phosphorylation potential. Our results showed that EGFR-Y845F mutant considerably reduced its ability of EGF-induced phosphorylation to its dimeric counterparts, suggesting the importance of Y845 transphosphorylation in this process.

The EGFR-Y845F mutant reduces its ability to stimulate cell growth, DNA synthesis and EGFR-mediated tumor growth. To determine the functional consequences of the loss of Y845 transphosphorylation, we used MCF7 stable clone cells to perform functional assays. The results showed that EGFRY845F mutant reduced its ability to respond to EFG-stimulated cell growth and DNA synthesis (Fig. 4). Furthermore, EGFRY845 mutant significantly reduced EGFR-mediated tumor growth in nude mice (Fig. 5), suggesting that transphosphorylation of EGFR-Y845 is critical to its biological function.

\section{Discussion}

In the present study, we clearly demonstrated the intrinsic correlation between EGFR transphosphorylation and autophosphorylation. Our results support that transphosphorylation of EGFR at Y845, which is located in its activation loop and a critical tyrosine transphosphorylation site, potentially influences its kinase activity and biological function. Upon the replacement of tyrosine to phosphorylation incapable phenylalanine, we observed that EGFR autophosphorylation and ligand-induced kinase activity were significantly reduced. Meanwhile, the ability of the EGFR-Y845F mutant 
A

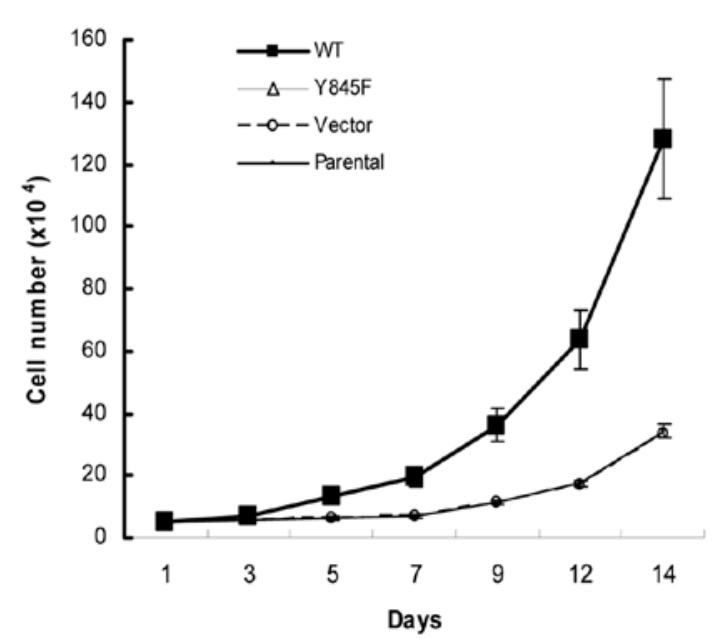

B

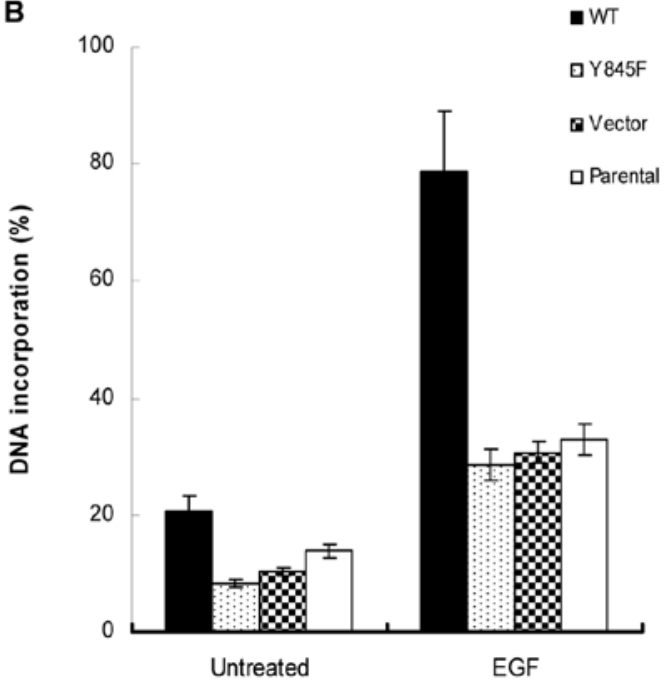

Figure 4. The EGFR-Y845F mutant reduces it ability to stimulate EGF-induced cell growth and DNA incorporation. MCF7 cells stably expressed either wt-EGFR or EGFR-Y845F were employed in the present study. (A) The cells were seeded into 6-well plates in the presence of EGF in triplicate and the cell number was counted at indicated time intervals. (B) The cells were seeded into 6-well plates in the presence of EGF in triplicate. The percentage of DNA incorporated cells was rated as described in Materials and methods. The results represent an average of three independent experiments as each experiment was set up in triplicate.

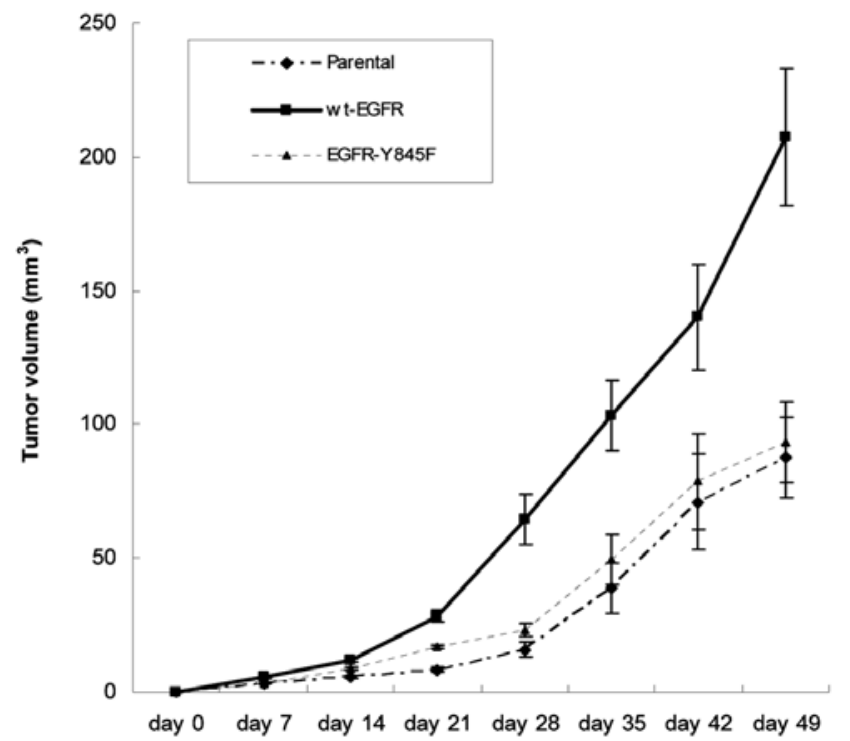

Figure 5. The EGFR-Y845F mutant reduces its ability to stimulate tumor cell growth in vivo. MCF7 cells stably expressed either wt-EGFR or EGFR-Y845F were inoculated into the nude mice. The tumor size was measured once per week as indicated in the image.

responding to EGF ligand stimulation was severely impaired (Figs. 3 and 4). The functional results are also consistent with this alteration as to which EGF-induced cell growth, DNA synthesis and tumor growth in nude mice were impaired as well (Figs. 4 and 5). A previous study demonstrated that EGFR-Y845 phosphorylation was essential for ligand-stimulated DNA synthesis. However, EGFR autophosphorylation seemed independent of its transphosphorylation at Y845 (27). In contrast to this report, one study demonstrated that EGFRY845F mutant actually augmented both ligand-stimulated EGFR tyrosine phosphorylation and DNA synthesis (28). We consider that these inconsistent observations may result from
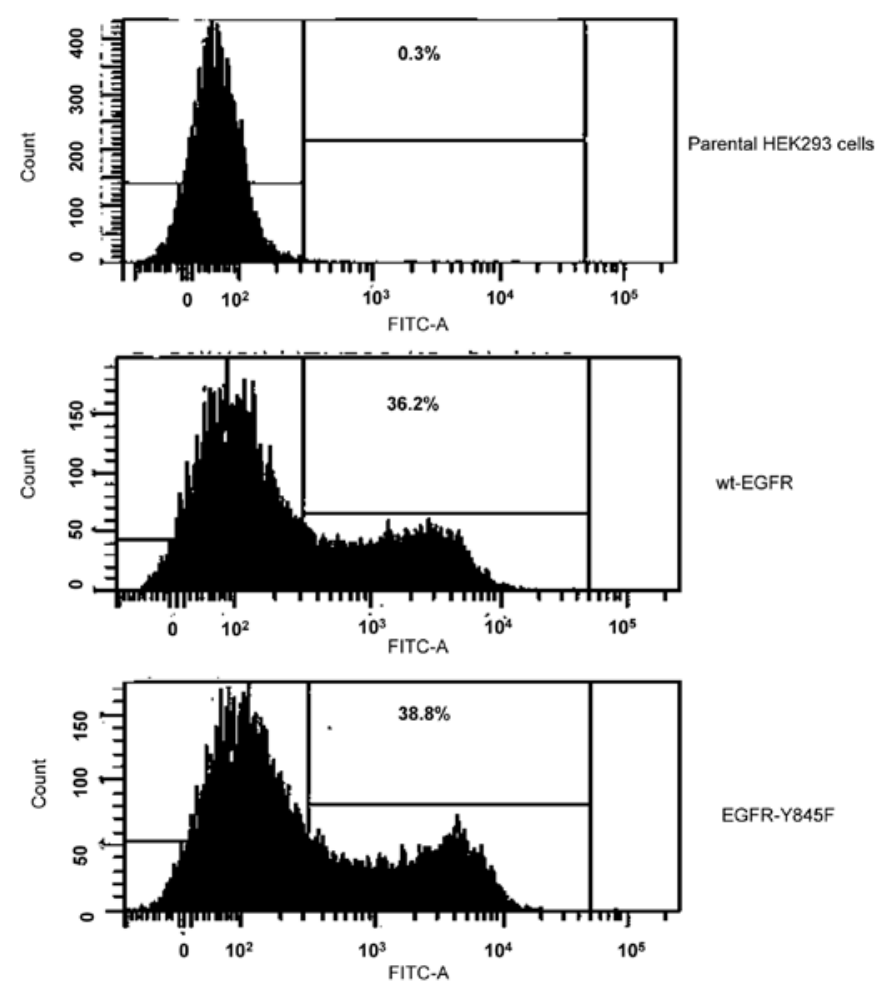

Figure 6. Comparison of cell surface expression and distribution of EGFR between wt-EGFR and EGFR-Y845F mutant in HEK293 cells. Either wt-EGFR or EGFR-Y845F was transiently expressed in HEK293 cells. The cells were immunostained with an anti-EGFR antibody and amplified with an FITC labeled anti-IgG antibody, then subjected to flow cytometry analysis.

different cell types and EGFR expression levels within the underlying cell lines. In addition, we observed that massively transient expression of EGFR in HEK293 cells, EGFR could be overphosphorylated in the absence of EGF ligand. 
Although the cells were subjected to $24 \mathrm{~h}$ serum starvation, both wt-EGFR and EGFR-Y845F tyrosine phosphorylation were still detectable (data not shown), suggesting that excessive EGFR molecules on the cell surface may be sufficient for triggering its tyrosine phosphorylation even without participation of its ligands. Notably, we observed that cell surface expression levels and distribution between wt-EGFR and EGFR-Y845 are very similar to each other (Fig. 6), suggesting the EGFR-Y845F mutant might not change its protein folding and structure. What caused EGFR phosphorylation and activation remains elusive as this phenomenon seemingly mimics some types of cancer in the clinical settings such as glioblastoma. Therefore, it is of interest to understand how this ligand independent EGFR phosphorylation occurs in the first place under the conditions of EGFR overexpression. To avoid this issue, we carefully selected cell lines with undetectable levels of endogenous EGFR and generated stable clones expressing appropriate amounts of either wt-EGFR or mutants as confirmed by flow cytometry analysis (data not shown). The final selected clones expressing EGFR or mutants with appropriate levels of EGFR that lacks self phosphorylation were employed in the present study. These clones allowed us to mimic EGFR cellular responses to its ligands in the conditions close to the physiological environment. Therefore, the results may possibly reflect real situations of EGFR phosphorylation and functions. Collectively, our results from these studies suggested that transphosphorylation of EGFR plays a critical role in its ligand-induced kinase activity and functions as demonstrated in Figs. 4 and 5.

As an important target therapeutic molecule in various types of cancer such as non-small cell lung, breast, colon and brain cancer, EGFR inhibition is dominant in the field of new drug development and clinical applications. The mutations of EGFR involved in tumor response or resistance to target therapeutic reagents in various types of cancer have been documented as well. These advances are helpful and critical to basic research and clinical settings. However, the mutation and activation of EGFR eventually have to link to its phosphorylation and kinase activity, especially the interplay of its intrinsic phosphorylation. Therefore, elucidation of the interaction of EGFR transphosphorylation and autophosphorylation not only provides insights into EGFR activation, but may also open up a novel venue towards EGFR-associated target therapy.

\section{Acknowledgements}

This study was supported by the National Natural Science Foundation of China NSFC 31271495 (to H.S.), and the Priority Academic Program Development of Jiangsu Higher Education Institutions (to L.Y.).

\section{References}

1. Herbst RS, Fukuoka M and Baselga J: Gefitinib - a novel targeted approach to treating cancer. Nat Rev Cancer 4: 956-965, 2004.

2. Hynes NE and Lane HA: ERBB receptors and cancer: the complexity of targeted inhibitors. Nat Rev Cancer 5: 341-354, 2005

3. Carlin CR and Knowles BB: Identity of human epidermal growth factor (EGF) receptor with glycoprotein SA-7: evidence for differential phosphorylation of the two components of the EGF receptor from A431 cells. Proc Natl Acad Sci USA 79: 5026-5030, 1982.
4. Lynch TJ, Bell DW, Sordella R, et al: Activating mutations in the epidermal growth factor receptor underlying responsiveness of non-small-cell lung cancer to gefitinib. N Engl J Med 350: 2129-2139, 2004

5. Sharma SV, Bell DW, Settleman J and Haber DA: Epidermal growth factor receptor mutations in lung cancer. Nat Rev Cancer 7: 169-181, 2007.

6. Verveer PJ, Wouters FS, Reynolds AR and Bastiaens PI: Quantitative imaging of lateral ErbB1 receptor signal propagation in the plasma membrane. Science 290: 1567-1570, 2000.

7. Lax I, Fischer R, Ng C, Segre J, Ullrich A, Givol D and Schlessinger J: Noncontiguous regions in the extracellular domain of EGF receptor define ligand-binding specificity. Cell Regul 2: 337-345, 1991.

8. Zhou M, Felder S, Rubinstein M, Hurwitz DR, Ullrich A, Lax I and Schlessinger J: Real-time measurements of kinetics of EGF binding to soluble EGF receptor monomers and dimers support the dimerization model for receptor activation. Biochemistry 32 : 8193-8198, 1993.

9. Sorokin A, Lemmon MA, Ullrich A and Schlessinger J: Stabilization of an active dimeric form of the epidermal growth factor receptor by introduction of an inter-receptor disulfide bond. J Biol Chem 269: 9752-9759, 1994.

10. Lemmon MA, Bu Z, Ladbury JE, et al: Two EGF molecules contribute additively to stabilization of the EGFR dimer. EMBO J 16: 281-294, 1997.

11. Klein P, Mattoon D, Lemmon MA and Schlessinger J: A structure-based model for ligand binding and dimerization of EGF receptors. Proc Natl Acad Sci USA 101: 929-934, 2004.

12. Dawson JP, Berger MB, Lin CC, Schlessinger J,Lemmon MA and Ferguson KM: Epidermal growth factor receptor dimerization and activation require ligand-induced conformational changes in the dimer interface. Mol Cell Biol 25: 7734-7742, 2005.

13. Burgess AW, Cho HS, Eigenbrot $\mathrm{C}$, et al: An open-and-shut case? Recent insights into the activation of EGF/ErbB receptors. Mol Cell 12: 541-552, 2003.

14. Zhang X, Gureasko J, Shen K, Cole PA and Kuriyan J: An allosteric mechanism for activation of the kinase domain of epidermal growth factor receptor. Cell 125: 1137-1149, 2006.

15. Zhang X, Pickin KA, Bose R, Jura N, Cole PA and Kuriyan J: Inhibition of the EGF receptor by binding of MIG6 to an activating kinase domain interface. Nature 450: 741-744, 2007.

16. Jura N, Endres NF, Engel K, et al: Mechanism for activation of the EGF receptor catalytic domain by the juxtamembrane segment. Cell 137: 1293-1307, 2009.

17. Honegger AM, Kris RM, Ullrich A and Schlessinger J: Evidence that autophosphorylation of solubilized receptors for epidermal growth factor is mediated by intermolecular cross-phosphorylation. Proc Natl Acad Sci USA 86: 925-929, 1989.

18. Skolnik EY, Margolis B, Mohammadi M, et al: Cloning of PI3 kinase-associated p85 utilizing a novel method for expression/cloning of target proteins for receptor tyrosine kinases. Cell 65: 83-90, 1991 .

19. Sordella R, Bell DW, Haber DA and Settleman J: Gefitinibsensitizing EGFR mutations in lung cancer activate anti-apoptotic pathways. Science 305: 1163-1167, 2004.

20. Nyati MK, Morgan MA, Feng FY and Lawrence TS: Integration of EGFR inhibitors with radiochemotherapy. Nat Rev Cancer 6: 876-885, 2006.

21. Sorkin A and Goh LK: Endocytosis and intracellular trafficking of ErbBs. Exp Cell Res 315: 683-696, 2009.

22. Citri A and Yarden Y: EGF-ERBB signalling: towards the systems level. Nat Rev Mol Cell Biol 7: 505-516, 2006.

23. Yarden Y and Sliwkowski MX: Untangling the ErbB signalling network. Nat Rev Mol Cell Biol 2: 127-137, 2001.

24. Yarden Y: The EGFR family and its ligands in human cancer: signalling mechanisms and therapeutic opportunities. Eur J Cancer 37 (Suppl 4): S3-S8, 2001.

25. Morgan S and Grandis JR: ErbB receptors in the biology and pathology of the aerodigestive tract. Exp Cell Res 315: 572-582, 2009.

26. Goel S, Hidalgo M and Perez-Soler R: EGFR inhibitor-mediated apoptosis in solid tumors. J Exp Ther Oncol 6: 305-320, 2007.

27. Tice DA, Biscardi JS, Nickles AL and Parsons SJ: Mechanism of biological synergy between cellular Src and epidermal growth factor receptor. Proc Natl Acad Sci USA 96: 1415-1420, 1999.

28. Gotoh N, Tojo A, Hino M, Yazaki Y and Shibuya M: A highly conserved tyrosine residue at codon 845 within the kinase domain is not required for the transforming activity of human epidermal growth factor receptor. Biochem Biophys Res Commun 186: 768-774, 1992. 